\title{
GASTRO-INTESTINAL STUDIES. I. GASTRIC JUICE IN PERNICIOUS ANEMIA
}

\author{
By O. M. HELMER, PAUL J. FOUTS, AND L. G. ZERFAS \\ (From the Lilly Laboratory for Clinical Research, Indianapolis City Hospital and the \\ Department of Medicine, Indiana University School of Medicine, Indianapolis)
}

(Received for publication June 20, 1932)

\section{GASTRIC JUICE IN PERNICIOUS ANEMIA}

Several authors have indicated that the determination of the hydrochloric acid, in itself, is not an adequate measure of the impairment of the secretory activity of the gastric mucosa. Michaelis (1) demonstrated this fact by the simultaneous determination of acid and enzymes in gastric juice from cases of low and high acidity and achylia. Polland and Bloomfield (2) stated that a low pepsin output is a more delicate index of gastric function than low acid values. Davies (3) similarly reported low pepsin values in the achylia of pernicious anemia.

Since the determination of the enzyme content of the gastric juice may be a valuable aid in the differential diagnosis of certain types of anemia, we are presenting the data that we have obtained in a series of pernicious anemia cases and normal controls. In a later paper data will be presented on secondary anemia and some obscure types of anemia.

In this series of experiments we have determined the gastric enzymes, pepsin and rennin, the free and total acid, the hydrogen ion concentration and the nitrogen, chloride, and phosphorus content, in order to determine as fully as possible the extent of the dysfunction of the gastric glands in the achylia gastrica occuring in patients with pernicious anemia.

\section{MATERIAL AND METHODS}

Ten young, healthy adults, who worked in the hospital and who had no evidence of disease, were used as controls in these experiments. All of the forty-seven patients with pernicious anemia were proven cases, and a more detailed description of them will be found later in the paper.

No food or drink was given to the subjects between the evening meal and the morning of the test. Early in the morning, the fasting contents were removed by means of a Rehfuss tube, after which $0.5 \mathrm{mgm}$. of histamine hydrochloride was injected. All patients developed a generalized erythema following the injection. Subsequent samples of the gastric juice were collected at twenty minute intervals over a period of eighty minutes, great care being taken to remove as completely as possible all of the gastric contents at each withdrawal. The patients were advised to take particular care not to swallow saliva.

To fourteen of the forty-seven patients with pernicious anemia, intravenous liver extract was given at the end of the sixty minute period, and two more 
samples of gastric juice were collected. Each specimen of gastric juice was measured in a graduated cylinder and was filtered through paper before using it for analysis. The presence or absence of bile, blood, and mucus was noted.

The $\mathrm{pH}$ was determined by means of the colorimetric method of Brown (4) using one drop of gastric juice, the free acid by Sahli's method using $1 \mathrm{cc}$. of juice, and the total acid on $1 \mathrm{cc}$. of juice, titrating with $\mathrm{N} / 100$ sodium hydroxide using phenolphthalein as an indicator. Chlorides were measured by Wilson and Ball's modification (5) of the Van Slyke method, and nitrogen was determined, using $0.5 \mathrm{cc}$. of gastric juice, by the gasometric method of Van Slyke (6).

Phosphorus was determined by Fiske and Subbarow's method (7). Onehalf $\mathrm{cc}$. of the samples of gastric juice obtained from patients with pernicious anemia were digested in pyrex test tubes graduated to a volume of $10 \mathrm{cc}$. Larger samples, depending upon the acidity, were used when the subjects were normals. When normal juice was precipitated with trichloracetic acid, the filtrate contained practically the same amount of phosphorus as the whole juice, whereas, with samples from patients with pernicious anemia, we were unable to get clear filtrates after trichloracetic acid precipitation, and, therefore, the total phosphorus was determined on the whole juice.

Pepsin was determined by the method of Koch and McMeekin (8)-a simple, rapid, and accurate method, for which only one cc. of gastric juice was required. The substrate employed in this method was coagulated egg white, which is particularly useful in the determination of pepsin in gastric juice, since trypsin is not able to act upon it to an appreciable extent.

The coagulated egg white was prepared as follows: a 20 per cent solution of Merck's powdered egg white (or albumin) was prepared and coagulated with heat by placing it in a boiling water bath for fifteen minutes, stirring vigorously throughout the coagulation period. The coagulated egg white was filtered through cheese cloth, and was broken up into small pieces by passing it through a fine meat chopper. It was then shaken with an excess of 0.3 per cent hydrochloric acid for two hours, at $40^{\circ} \mathrm{C}$., to remove inorganic salts, colored extractives, and other substances which are soluble in 0.3 per cent hydrochloric acid, thereby reducing the soluble nitrogen blank of the egg white. The coagulated egg white was filtered through gauze with the aid of suction and was thoroughly washed with distilled water. It was then spread out on trays and dried in a current of warm air. After drying, it was ground so as to pass through an eighty mesh sieve.

To determine the amount of peptic activity, 4 grams of egg white were suspended in $99 \mathrm{cc}$. of 0.3 per cent hydrochloric acid in a $125 \mathrm{cc}$. Erlenmeyer flask, at room temperature. Then $1 \mathrm{cc}$. of gastric juice was added. The solution was shaken and placed in an incubator at $40^{\circ} \mathrm{C}$. for two hours where it was rotated vertically during the entire period of incubation. ${ }^{1}$ After the two hour incubation period, the solution was filtered by decantation through $18 \mathrm{~cm}$. quantitative filter paper. The refractive index was determined at $25^{\circ} \mathrm{C}$. on the filtrate by means of an emersion refractometer, and the readings were converted into "milligrams of pepsin" ( 1 to 4,000$)$ by means of a table prepared by Koch and McMeekin (8). Since the concentration of pepsin is so low in gastric juice obtained from patients with pernicious anemia, only two grams of egg white were used as a substrate, and the total volume of acid was made up to $50 \mathrm{cc}$. instead of $100 \mathrm{cc}$. Otherwise, the method was identical with that used with normal juice.

1 The water bath and shaker, designed by Doctor Koch, was obtained from the Arthur H. Thomas Company, Philadelphia. 
Rennin was determined by a method similar to the one described by Michaelis (1). Forty-five cc. of fresh, raw, cows' milk and 5 cc. of a 10 per cent solution of crystalline calcium chloride were mixed. Test tubes containing $2.5 \mathrm{cc}$. of this reagent were placed in the water bath at $40^{\circ} \mathrm{C}$., and, when they had attained the temperature of the bath, gastric juice equivalent to $1 / 100 \mathrm{cc}$. to $1 \mathrm{cc}$., depending upon the rennit activity of the juice, was added to the tubes and the clotting time noted. The rennit activity was converted into "milligrams of rennin" by referring to a table made by using the above technique on known concentrations of dried commercial rennin. The clotting time with the known concentrations of rennin are shown in Table 1.

TABLE 1

The clotting time required for known amounts of dry commercial rennin

$\begin{array}{ccc}\begin{array}{c}\text { Rennin } \\ \text { mgm. }\end{array} & \begin{array}{c}\text { Average clotting time } \\ \text { seconds }\end{array} & \begin{array}{c}\text { Average clotting time } \\ \text { minutes }\end{array} \\ 1.5 & 9 & \\ 1.0 & 10.5 & \\ 0.5 & 21 & \\ 0.3 & 31 & \\ 0.2 & 44 & \\ 0.1 & 73 & \\ 0.075 & 98 & \\ 0.050 & 145 & 1.63 \\ 0.030 & 222 & 3.70 \\ 0.020 & 334 & 5.90 \\ 0.010 & 650 & 10.83\end{array}$

RESULTS IN NORMAL SUBJECTS

In Table 2 are tabulated the results of the analyses of the gastric juice obtained from ten normal subjects.

In Chart I, the values obtained in an average normal subject are plotted. With the exception of one case, the fasting samples contained only a trace of mucus. After histamine stimulation, the juice became more watery, filtering with the greatest rapidity at the height of the acid secretion. The greatest volumes of juice were obtained at the twenty minute period and ranged from 35 to $80 \mathrm{cc}$., with an average of $56 \mathrm{cc}$.

In the fasting samples the $\mathrm{pH}$ varied from 1.6 to 7.2. The peak of the acid secretion was in the forty minute period in the majority of cases, with $\mathrm{pH}$ values of 1.1 or less. The free acid at its peak ranged from 72 to $140 \mathrm{cc}$. N/10 per $100 \mathrm{cc}$., and the total acid varied from 77 to 149 .

The pepsin and rennin were secreted at the same rate, with the peak at twenty minutes. At the height of secretion the pepsin varied from 1.4 to $7.1 \mathrm{mgm}$. per cc., with an average of $4.6 \mathrm{mgm}$., and the rennin varied from 15 to $70 \mathrm{mgm}$. The concentration of enzymes in the gastric juice did not follow the curve of acid secretion.

The nitrogen concentration fell after stimulation, which is in accord with the data in the literature. The lowest values for nitrogen were found at the peak of the acid secretion. The curve of the nitrogen concentration did not follow that of the enzymes. This is contrary to the findings of Polland and Bloomfield (9), who stated that in normal persons the con- 


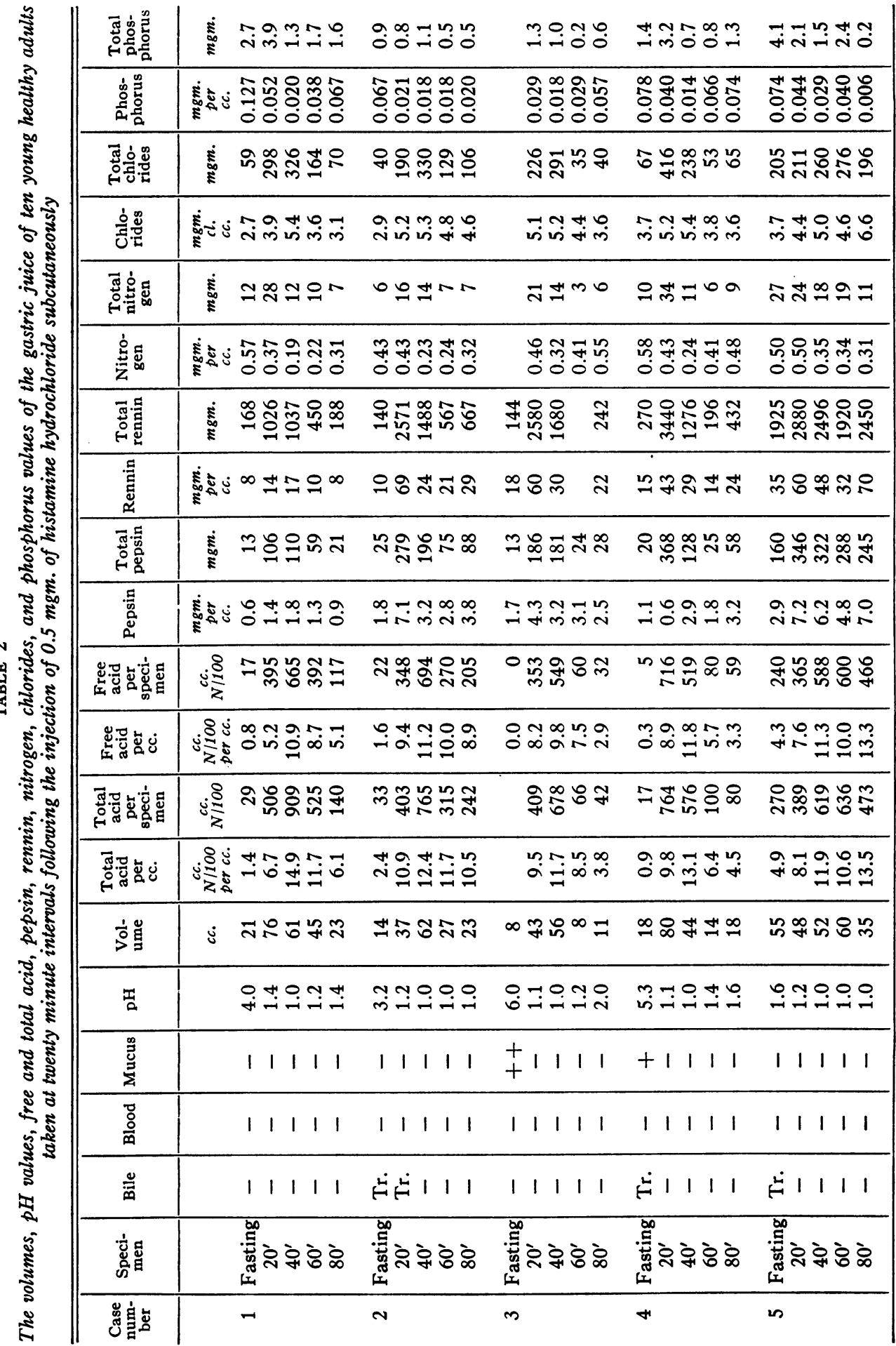


O. M. HELMER, PAUL J. FOUTS AND L. G. ZERFAS

\begin{tabular}{|c|c|c|c|c|c|}
\hline 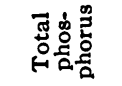 & है & 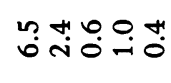 & Nim & 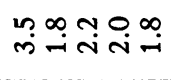 & 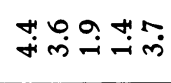 \\
\hline 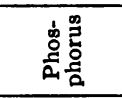 & 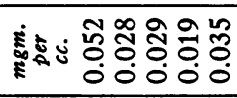 & 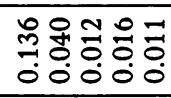 & 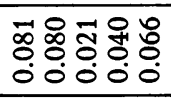 & 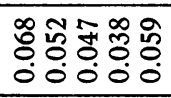 & 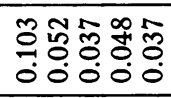 \\
\hline 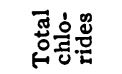 & : & 声있유융융 & 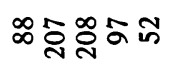 & 車을유ㅇㅛㅠ & 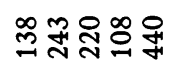 \\
\hline 完总 & 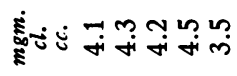 & ن & 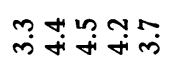 & 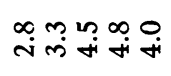 & $\ddot{m} \ddot{m} \ddot{m} \dot{m}$ \\
\hline 预㤩 & Еే & ลิล $=ニ \infty$ & $\stackrel{\sim}{\sim} \approx \approx a$ & $\stackrel{\infty}{\infty} \stackrel{\infty}{\sim} \stackrel{\infty}{-}$ 이 & 류 \\
\hline 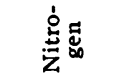 & 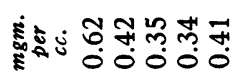 & 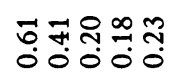 & 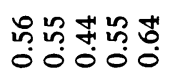 & 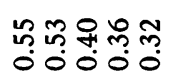 & 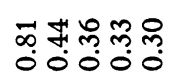 \\
\hline 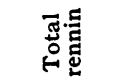 & 롫 & 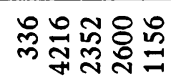 & 舟递음 & 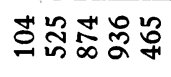 & 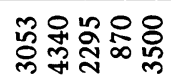 \\
\hline 曹 & ถ્心 & 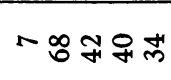 & 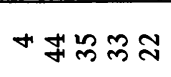 & $N \cong 9 \infty$ & 중요 \\
\hline 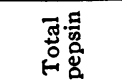 & 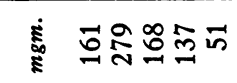 & 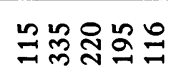 & 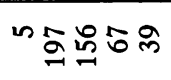 & 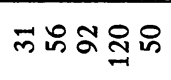 & 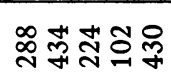 \\
\hline 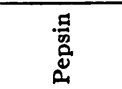 & 密气心 & 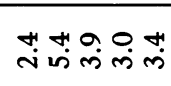 & 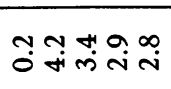 & نِ & 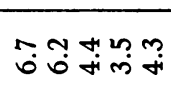 \\
\hline 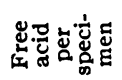 & 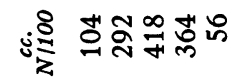 & 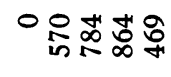 & 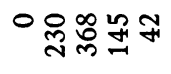 & 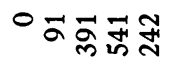 & 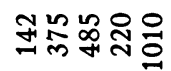 \\
\hline 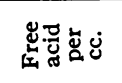 & ن웜 & 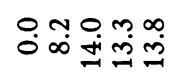 & 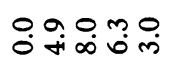 & O요유 & min \\
\hline 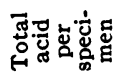 & 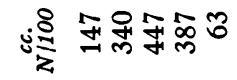 & 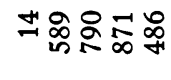 & 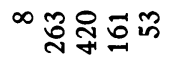 & 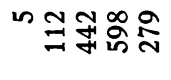 & 总蕃的志并 \\
\hline 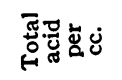 & نَّ & 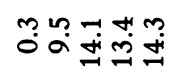 & 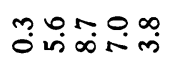 & பำ & 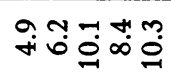 \\
\hline 递害 & 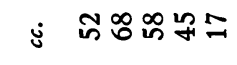 & 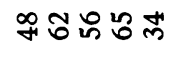 & ヘチ์ํㅀ & 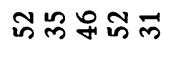 & 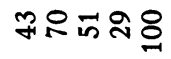 \\
\hline 垔 & 空苟 & ن요 &  &  & 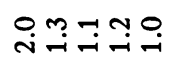 \\
\hline 惢 & $\begin{array}{lllll}1 & 1 & 1 & 1 & 1\end{array}$ & +1111 & $\begin{array}{lllll}1 & 1 & 1 & 1 & 1\end{array}$ & +1111 & $\begin{array}{lllll}1 & 1 & 1 & 1 & 1\end{array}$ \\
\hline$\frac{\overline{0}}{\square}$ & $\begin{array}{lllll}1 & 1 & 1 & 1 & 1\end{array}$ & $\begin{array}{lllll}1 & 1 & 1 & 1 & 1\end{array}$ & $\begin{array}{lllll}1 & 1 & 1 & 1 & 1\end{array}$ & $\begin{array}{lllll}1 & 1 & 1 & 1 & 1\end{array}$ & $\begin{array}{lllll}1 & 1 & 1 & 1 & 1\end{array}$ \\
\hline 芦 & $+\dot{\vec{H}} \dot{H}+\dot{H}$ & $\dot{H} 1111$ & 11111 & $\dot{H} \mid \begin{array}{lll}\mid & \dot{H}\end{array}$ & $\begin{array}{lllll}1 & 1 & 1 & 1 & 1\end{array}$ \\
\hline 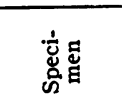 & 起兽 & 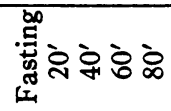 & . & 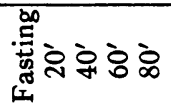 &  \\
\hline 惢家官 & 0 & $r$ & $\infty$ & $a$ & 으 \\
\hline
\end{tabular}


centration of pepsin falls markedly after stimulation and follows closely the curve of nitrogen concentration. In all but one of our normal individuals, there was an increase in enzyme concentration after histamine stimulation, whereas the nitrogen values were always lowered. The

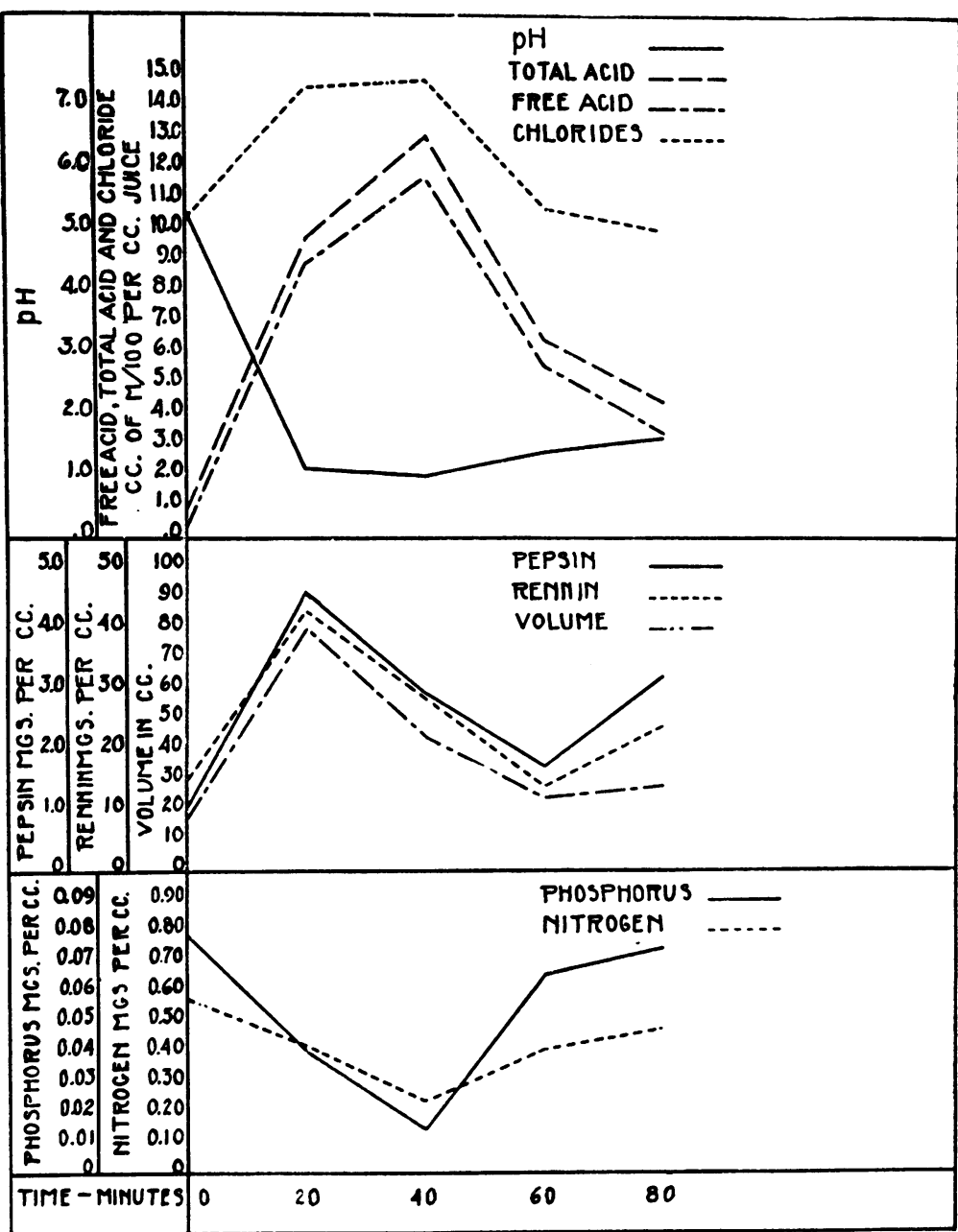

Values from Gastric Juice of Normal Subject Number 4 (a Typical Normal) Following the Subcutaneous Injection of 0.5 Mgm. of Histamine HydROCHLORIDE

secretion of enzymes apparently influenced the nitrogen concentration in Case 2, Case 5, and Case 8; after stimulation, there was the usual increase in acid, with a simultaneous increase in pepsin and rennin of greater proportions than usual, but with a comparatively smaller decrease in nitrogen concentration. 
The total chloride concentration, in general, followed the acid concentration; however, the increase in chlorides was not in proportion to the change in acidity, and could not, in itself, account for the increased acidity. Gamble and McIver (10) and Austin and Gammon (11), using dogs with gastric pouches, stated that the chloride concentration remained almost constant during their experiments.

The total phosphorus concentration decreased as the acidity increased, reaching its low point at the height of the acid secretion. As the acid decreased, the phosphorus values returned to values approaching those of the fasting samples. Bulger, Stroud, and Heideman (12) point out that, if much acid is produced, the phosphates decrease, and they attribute the fluctuations to relative amounts of secretion from the mucous glands. We have also found that if stimulation did not produce a secretion of free hydrochloric acid, the concentration of phosphate often increased. Hoesch (13), too, showed that the phosphorus content was low when the acidity was high, and, in cases of anacidity or achylia, the phosphorus content of the gastric juice was high. Hoesch believes that the antagonism between chloride and phosphate may be an important factor in the secretory mechanism that produces free hydrochloric acid.

Bolton and Goodhart (14) believe that mucus is an important factor in controlling the acidity of the stomach, especially when there is only a small flow of gastric juice. Since the nitrogen and phosphorus of the gastric juice are probably secreted mainly by the mucous glands, the fall in both nitrogen and phosphorus with active acid secretion combined with their subsequent rise as the acid secretion diminishes, might further point to an important rôle of gastric mucus in the control of the acidity of the stomach.

\section{RESULTS IN PERNICIOUS ANEMIA}

Table 3 records the results of the forty-seven individual analyses, while Chart II shows the average of the results obtained in the fortyseven cases of pernicious anemia. The gastric juice obtained in patients with pernicious anemia was usually small in volume and contained much mucus, but the samples with the greatest volumes usually contained the least mucus. Vineburg and Babkin (15), and Gilman and Cowgill (16) stated that histamine did not stimulate the gastric enzymes but that pilocarpine did. We did separate, complete gastric analyses on one patient, after $0.5 \mathrm{mgm}$. histamine given subcutaneously, after $3.2 \mathrm{mgm}$. pilocarpine subcutaneously, and after $20 \mathrm{cc}$. of liver extract intravenously. There was practically no difference in the three analyses, so we considered histamine an adequate stimulus. Two patients also had gastric analyses after histamine stimulation, first while in relapse and again soon after remission had been induced by liver extract, and there were no differences in the gastric findings. 


\begin{tabular}{|c|c|c|c|c|c|}
\hline 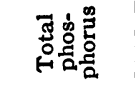 & है & & & & \\
\hline 突总 & हैं & & & & \\
\hline 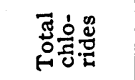 & نे نَ & & & & \\
\hline 它密苋 & हैछ & & & & \\
\hline 可题 & 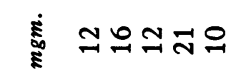 & $N-\sin ^{\circ}$ & 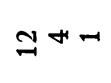 & $\operatorname{san}$ & $\infty \stackrel{+}{+} \vec{\sim}$ \\
\hline 产莣 & نَ & 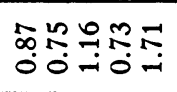 & 角 & 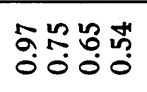 & 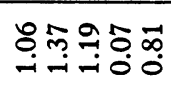 \\
\hline 苨 & & & & & \\
\hline 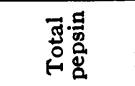 & 空 & 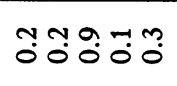 & 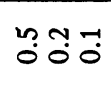 & 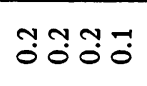 & ఝ̃ \\
\hline 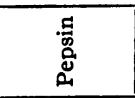 & 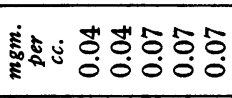 & 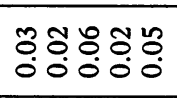 & 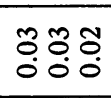 & 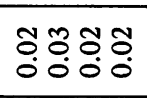 & 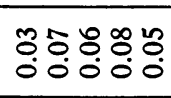 \\
\hline 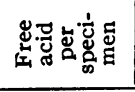 & $\dot{8} \frac{8}{z} 00000$ & 00000 & 000 & 0000 & 00000 \\
\hline 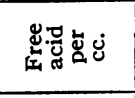 & $8: 00000$ & 00000 & 000 & 0000 & 00000 \\
\hline 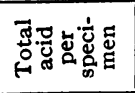 & $\dot{0} \frac{8}{z}-\infty$ in \pm 0 & 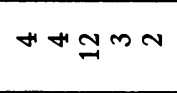 & $m-1$ & in $\rightarrow$ & ーシュサむ \\
\hline نำ & ن & חִ & 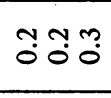 & ஸั & 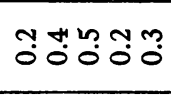 \\
\hline 官苛 & $\dot{s} \pm 0$ & $\infty a \exists$ オ & 우 & Ninn in & 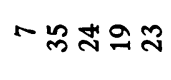 \\
\hline 公 & 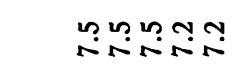 & 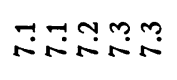 & مִ & $\begin{array}{llll}\sim & \infty & 0 & 0 \\
\infty & \infty & \infty & \infty \\
\infty & \infty & \infty\end{array}$ & ن \\
\hline 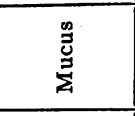 & $\begin{array}{l}+ \\
+ \\
+\end{array}$ & $+++\dot{+}+$ & $\begin{array}{l}++ \\
++ \\
++\end{array}$ & $\begin{array}{l}++++ \\
++++ \\
+\end{array}$ & $\begin{array}{l}++ \\
+ \\
+\end{array}++$ \\
\hline$\frac{\square}{0}$ & $++++t$ & $1++1+$ & 111 & $11++$ & $11++1$ \\
\hline 气्ञ & $111++$ & $11+11$ & 111 & 1111 & $11+11$ \\
\hline 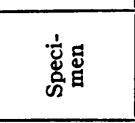 & 蜩 & 营 & . & 总 & 总 \\
\hline 总㹸㟔 & - & $N$ & $m$ & $H$ & in \\
\hline
\end{tabular}


O. M. HElmer, PAUl J. FOUTS AND L. G. ZERFAS

\begin{tabular}{|c|c|c|c|c|c|}
\hline 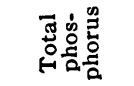 & $\stackrel{\Sigma}{\dot{\Sigma}}$ & & & & $\ddot{m} \stackrel{0}{i} \vec{i}$ \\
\hline 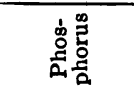 & हैं & & & & 쿵우웅 \\
\hline 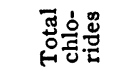 & نَ & & & $\approx 0$ & 리요묘 \\
\hline 放: & $\dot{E}$ & & & $\stackrel{\infty}{\rightarrow} \stackrel{9}{-}$ & $\stackrel{\infty}{\text { in }}$ \\
\hline 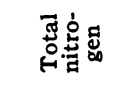 & Еี & $m+N N$ & & H m & 음 욤수 \\
\hline$\stackrel{\dot{\dot{g}}}{\mathrm{z}}$ & 葛: & भ̊心 & & ণั & 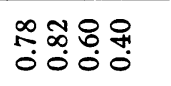 \\
\hline 竞 & & & & & \\
\hline 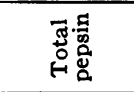 & 音 & 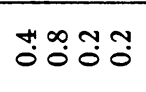 & 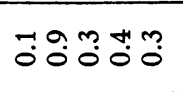 & 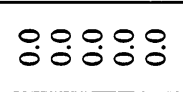 & ㅂㅇㅇㅇㅇㅇㅇㅇㅇ \\
\hline 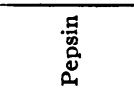 & 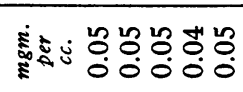 & ஜோல் & 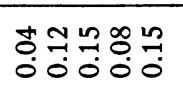 & 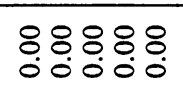 & $\begin{array}{l}0.8880 \\
00000 \\
00000\end{array}$ \\
\hline 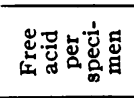 & $i \frac{8}{z} 00000$ & 0000 & 00000 & 00000 & 00000 \\
\hline 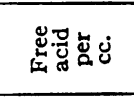 & ن⿺辶寸 & 0000 & 00000 & 00000 & 00000 \\
\hline 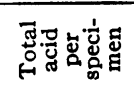 & $i \frac{8}{z} a n a m+$ & min & & & $a ㅇ ㅡ$ \\
\hline ن & 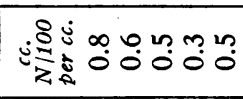 & ఫั: & & & 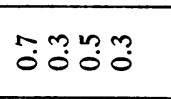 \\
\hline 市泀 & $\dot{\cup} \approx \approx \infty \circ \infty$ & $\infty \pm$ in in & $m N N \ln$ & nont m m in & m \\
\hline 公 & بْ به & 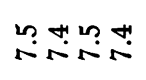 & $\stackrel{\infty}{\sim} \stackrel{\infty}{\sim} \stackrel{\infty}{\sim}$ & 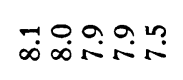 & 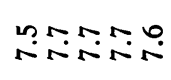 \\
\hline$\sum_{\Sigma}^{m}$ & $\stackrel{+}{+}+\stackrel{+}{++}$ & +++ & $+t+11$ & 11111 & $11++1$ \\
\hline के & 11111 & 1111 & $+1+++$ & $\begin{array}{lllll}1 & 1 & 1 & 1 & 1\end{array}$ & $1111+$ \\
\hline 苗 & ++111 & 1111 & $\begin{array}{lllll}1 & 1 & 1 & 1 & 1\end{array}$ & ++111 & $+\dot{E} 111$ \\
\hline $\begin{array}{l}\text { 它: } \\
\text { 遌 }\end{array}$ &  & . & 总 & 总 & 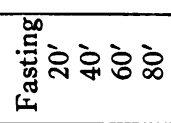 \\
\hline 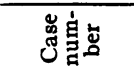 & 0 & $n$ & $\infty$ & $a$ & 음 \\
\hline
\end{tabular}




\begin{tabular}{|c|c|c|c|c|}
\hline 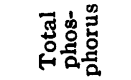 & : & & & ํㅜㄹํํ \\
\hline 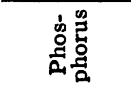 & : & & & 娄: \\
\hline 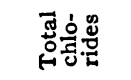 & 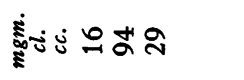 & $\bar{\sim} ヘ \stackrel{N}{N}$ & 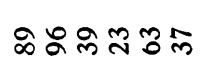 & 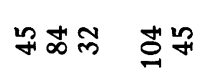 \\
\hline 爮密 & 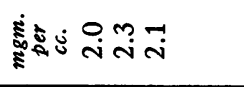 & $\vec{i} \stackrel{\infty}{-\infty} \stackrel{\infty}{i}$ & mָ̃ & 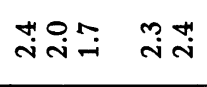 \\
\hline 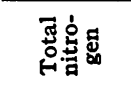 & 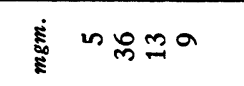 & $m \pm \sigma \infty$ & 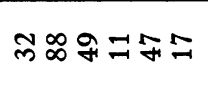 & $\ddot{\sim}$ \\
\hline 芶范 & हن் & 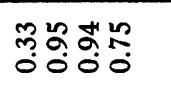 & ‡ & 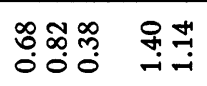 \\
\hline 范 & $\dot{H}$ & 00000 & 00000 & $\dot{\leftrightarrow}$ \\
\hline 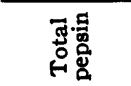 & : & ஜฺ̊ & 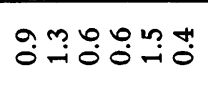 & 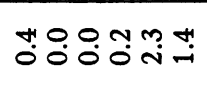 \\
\hline 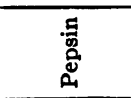 & 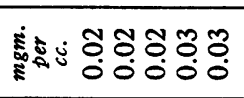 & 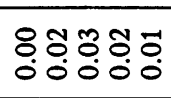 & 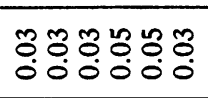 & 중유유. \\
\hline 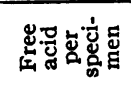 & $4 \frac{8}{2} 00000$ & 00000 & 000000 & 000000 \\
\hline ن & نs: & 00000 & 000000 & 000000 \\
\hline 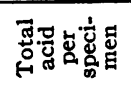 & $\dot{8} \frac{8}{z}$ Non-mm & Nintin & ○ & $0+N-a \infty$ \\
\hline 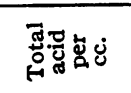 & ن⿺辶ِ & ஸ̃ற & 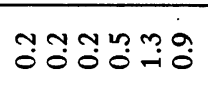 & 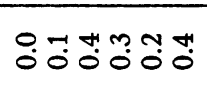 \\
\hline 宁首 & 의 $\infty$ 아푬으 & 으느으음 & 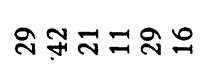 & 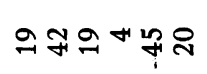 \\
\hline 窘 & 员 & 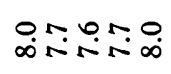 & 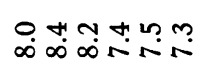 & ஸุஷ \\
\hline 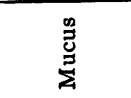 & +1111 & $\begin{array}{l}+++++ \\
++++\end{array}$ & $111+11$ & 1111111 \\
\hline : & $1111+$ & $111+1$ & ++++11 & $\begin{array}{llllll}1 & 1 & 1 & 1 & 1 & 1\end{array}$ \\
\hline 苛 & 11111 & $\begin{array}{lllll}1 & 1 & 1 & 1 & 1\end{array}$ & $+\dot{H} \dot{E} 1+\dot{H}$ & $\begin{array}{llllll}1 & 1 & 1 & 1 & 1 & 1\end{array}$ \\
\hline $\begin{array}{l}\text { 它: } \\
\text { 幽 }\end{array}$ & 营 & 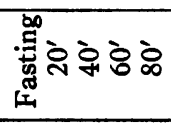 & 总 & 苞灾 \\
\hline Uू & $\exists$ & $\approx$ & $m$ & 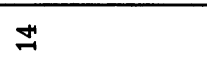 \\
\hline
\end{tabular}




\begin{tabular}{|c|c|c|c|c|}
\hline 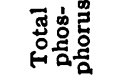 & مُด & $\stackrel{\infty}{-i} \underset{i}{\stackrel{0}{i}} \stackrel{\infty}{-}$ & mָָ & 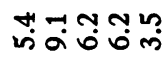 \\
\hline 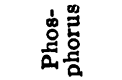 & 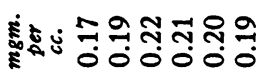 & 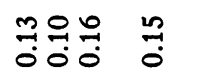 & 궁: 뭉 & 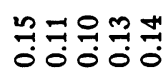 \\
\hline 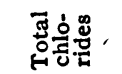 & 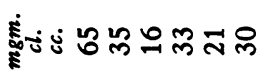 & 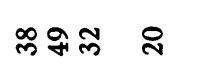 & 이륭무 & 음윰유융 \\
\hline 完总 & 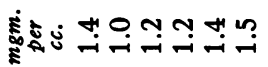 & సiํ요 & $\stackrel{\infty}{i} \stackrel{\infty}{i} \stackrel{0}{m}$ & 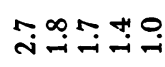 \\
\hline 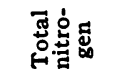 & 퐁 & 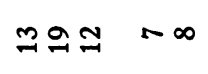 & ดテลㅇำ & 옹워 \\
\hline 总品 & E్ & 尽ニ & 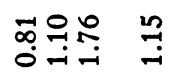 & 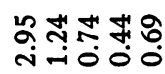 \\
\hline 运 & 000 & 0 & O $\dot{H} \dot{H}$ & 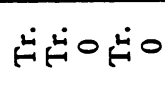 \\
\hline 需 & E⿱宀㠯心 & 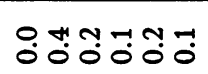 & 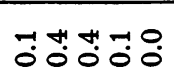 & 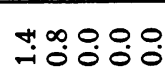 \\
\hline 咅 & 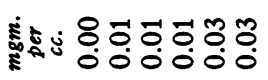 & 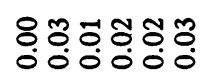 & 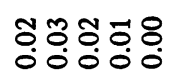 & 苛8:80 \\
\hline 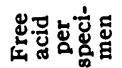 & ن: $\frac{8}{z} 000000$ & 000000 & 00000 & 00000 \\
\hline ن & $\dot{8} \frac{8 \dot{0}}{z} 000000$ & 000000 & 00000 & 00000 \\
\hline 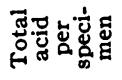 & $\dot{8} \frac{8}{z} a n-\approx 0 \infty$ & mina 0 & otom & 00000 \\
\hline 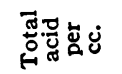 & ن & 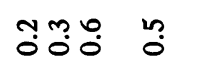 & 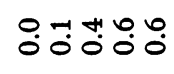 & 웅ㅇㅇㅇㅇ \\
\hline 官苜 & ن் & $\sharp ニ 0 m \simeq n$ & $=\operatorname{son} \cos a$ & 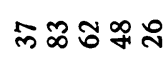 \\
\hline 䛱 & 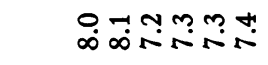 & 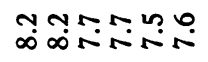 & 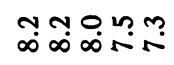 & 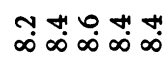 \\
\hline 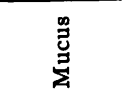 & . $\dot{\uplus} 11111$ & $\begin{array}{l}++ \\
++\end{array}$ & 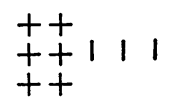 & 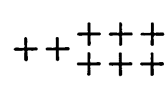 \\
\hline 苂 & $\begin{array}{llllll}1 & 1 & 1 & 1 & 1 & 1\end{array}$ & 点出 $1+++$ & $111+1$ & $\begin{array}{lllll}1 & 1 & 1 & 1 & 1\end{array}$ \\
\hline 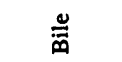 & $\begin{array}{llllll}1 & 1 & 1 & 1 & 1 & 1\end{array}$ & $\stackrel{\dot{H}}{1} 1 \quad 1 \quad 1 \quad 1 \quad 1$ & $11++\dot{E}$ & $\begin{array}{l}+ \\
+\end{array}$ \\
\hline 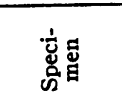 & 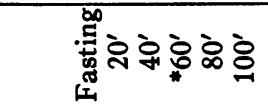 & 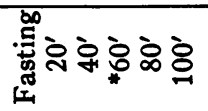 & 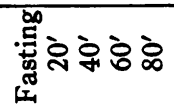 & 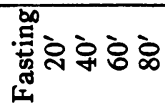 \\
\hline 岕富导 & 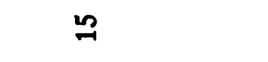 & $\stackrel{0}{\sim}$ & $\approx$ & $\stackrel{\infty}{\sim}$ \\
\hline
\end{tabular}




\begin{tabular}{|c|c|c|c|c|c|}
\hline 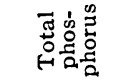 & 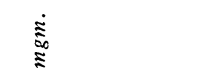 & & & & 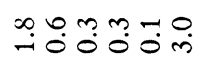 \\
\hline 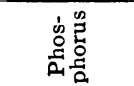 & 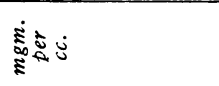 & & & & 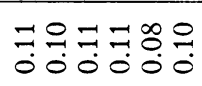 \\
\hline 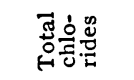 & ن & & & & 구 \\
\hline 递苋 & 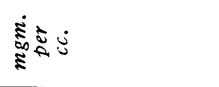 & & & & $\stackrel{n}{-}$ \\
\hline 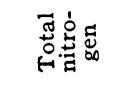 & $\dot{\Sigma}$ & & & & \\
\hline 苞范 & 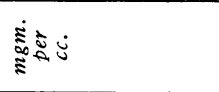 & & & & \\
\hline 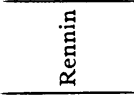 & 㟔出苫 & & $00 \stackrel{\leftrightarrow}{H}$ & & 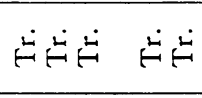 \\
\hline 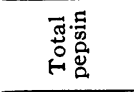 & : & 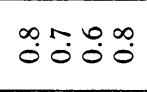 & ํํำ & 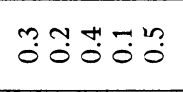 & 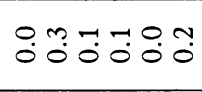 \\
\hline 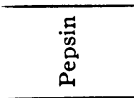 & 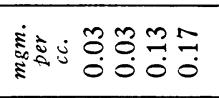 & 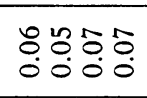 & 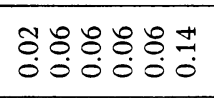 & 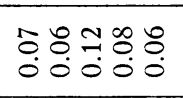 & $\begin{array}{l}8: 000 \% \\
000000 \\
000\end{array}$ \\
\hline 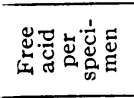 & $i_{i}^{8} 0000$ & 0000 & 000000 & 00000 & 000000 \\
\hline 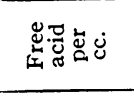 & 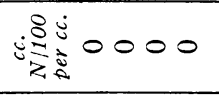 & 0000 & 000000 & 00000 & 000000 \\
\hline 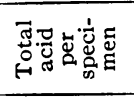 & $\underset{ن}{3} \stackrel{8}{2}$ & & th in & & \\
\hline نं & 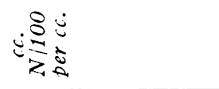 & & $\ddot{0} \quad \stackrel{0}{0}$ & & \\
\hline 官葸 & is $a+t i n$ & $\pm m \infty=$ & aningar & $n+\infty-\infty$ & $\approx 0 \sim \sim \underset{N}{ }$ \\
\hline 窝 & 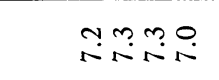 & $\vec{\infty} \underset{\infty}{0} \underset{\infty}{\infty} \underset{\infty}{\infty}$ & 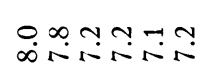 & 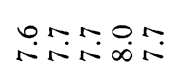 & 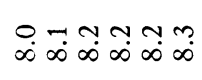 \\
\hline 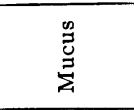 & 1111 & $\begin{array}{r}++ \\
++\end{array}$ & +++111 & 11111 & $111+11$ \\
\hline : & 1111 & $11++$ & $11111+$ & $1++11$ & $\begin{array}{llllll}1 & 1 & 1 & 1 & 1 & 1\end{array}$ \\
\hline 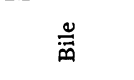 & $\begin{array}{l}++++ \\
+++\end{array}$ & $\begin{array}{l}++\dot{H} \\
++\dot{H}\end{array}$ & $111+\dot{E} 1$ & $1+11+$ & $1111++$ \\
\hline $\begin{array}{l}\text { 岀: } \\
\text { 遌 }\end{array}$ & 总 & 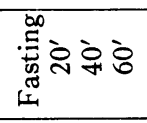 & 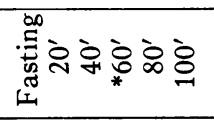 & 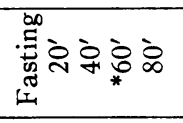 & 营 \\
\hline 总宗志 & 2 & 요 & $\vec{\sim}$ & $\tilde{ก}$ & $\ddot{\sim}$ \\
\hline
\end{tabular}


O. M. HELMER, PAUL J. FOUTS AND L. G. ZERFAS

\begin{tabular}{|c|c|c|c|c|c|}
\hline 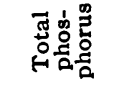 & $\dot{\Sigma}$ & $\stackrel{n}{n}$ & 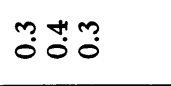 & 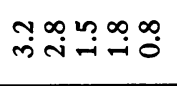 & ח̊ \\
\hline 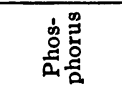 & ن & $\underset{0}{\dddot{1}} \div$ & ஜํㅇㅇㅠ & 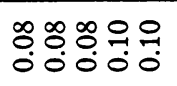 & 윰윰 \\
\hline 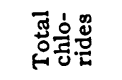 & نَ & జి 요 & & 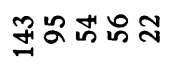 & \\
\hline 递苋 & ن & $\stackrel{n}{\sim} \stackrel{4}{i}$ & & 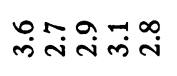 & \\
\hline 矛: & हू & $\dddot{7}$ & & ตேシ & \\
\hline 它总 & ن & ○ِّ & & 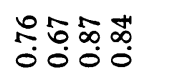 & \\
\hline 苞 & 00 & 0 & ثं० 0 & $\dot{H} \circ \circ \dot{H}$ & 00000 \\
\hline 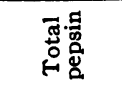 & : 0 : & 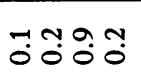 & تص & $\ddot{m} \vec{i} \underset{i}{n}$ & 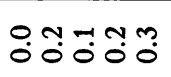 \\
\hline $\begin{array}{l}.5 \\
0 \\
0 \\
0 \\
0\end{array}$ & : & 훙훙웅영 & 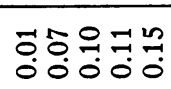 & $\begin{array}{l}8.0 \% \\
0000 \\
0000\end{array}$ & 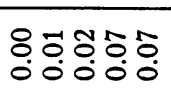 \\
\hline 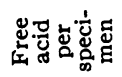 & ن⿺辶 0000 & 0000 & 00000 & 00000 & 00000 \\
\hline 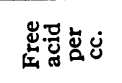 & ن $\frac{8 \dot{3}}{2} 0000$ & 0000 & 00000 & 00000 & 00000 \\
\hline 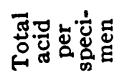 & $\dot{3} \frac{8}{z}$ & & & DOONO & \\
\hline 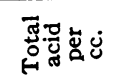 & ن & & & 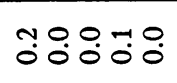 & \\
\hline 官茎 & $\dot{s}=m+\alpha$ & mבan & nansm & 우융요 & O름 \\
\hline 公 & 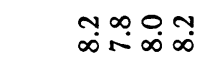 & 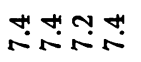 & بْ & 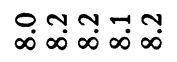 & 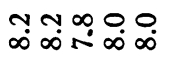 \\
\hline 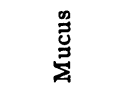 & $++\underset{+}{++}$ & $\begin{array}{l}+++ \\
++t\end{array}$ & $\begin{array}{lllll}1 & 1 & 1 & 1 & 1\end{array}$ & 111111 & $+1+11$ \\
\hline$\frac{\circ}{0}$ & 1111 & 1111 & $1++++$ & 111111 & 111111 \\
\hline 䒢 & 1111 & 1111 & $\dot{H} 1 \quad 1 \quad 1 \quad 1$ & $\begin{array}{lllll}1 & 1 & 1 & 1 & 1\end{array}$ & $111+_{+}^{+}$ \\
\hline 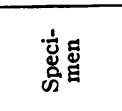 & 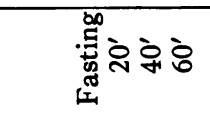 & 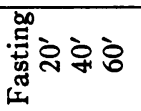 & 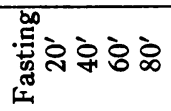 & 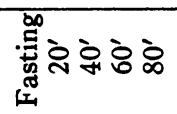 & 选点 \\
\hline 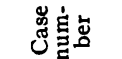 & ম & $\stackrel{2}{2}$ & $\stackrel{\sim}{\sim}$ & $\hat{\sim}$ & $\stackrel{\infty}{\sim}$ \\
\hline
\end{tabular}




\begin{tabular}{|c|c|c|c|c|c|}
\hline 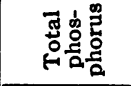 & 密 & - & 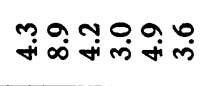 & 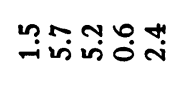 & సุo \\
\hline 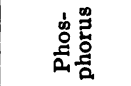 & : & $\frac{m}{0}$ & 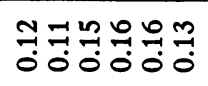 & 궁듕궁웅 & 䓍: \\
\hline 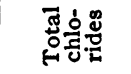 & 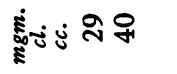 & ลี & 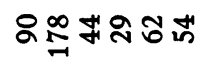 & 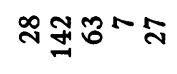 & \\
\hline 完总 & 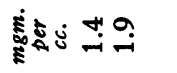 & $\stackrel{9}{-} \stackrel{\infty}{-1}$ & 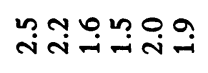 & 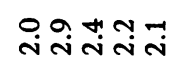 & \\
\hline 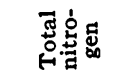 & ह & 으 & 沉 & 우암 & \\
\hline 豈范 & 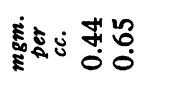 & $\stackrel{2}{0}$ & 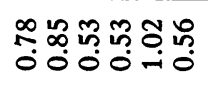 & 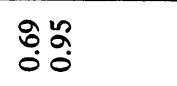 & \\
\hline 竭 & 000 & O岀 & ثं०00म̈० & $00 \dot{H}$ & $0 \dot{H}$ \\
\hline 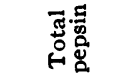 & 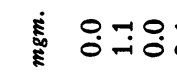 & 家 & نِ & 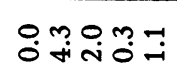 & 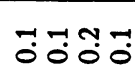 \\
\hline 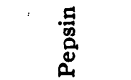 & 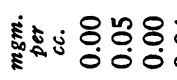 & : & 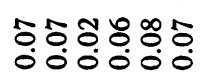 & 8: 0000 & 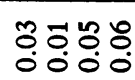 \\
\hline 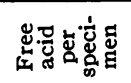 & $8 \frac{8}{z} 000$ & 00 & 000000 & 00000 & 0000 \\
\hline ن & ن & 00 & 000000 & 00000 & 0000 \\
\hline 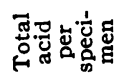 & is & 0 & 000000 & $000-$ & \\
\hline ن & 造 & $\ddot{0}$ & 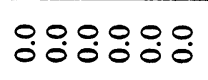 & $\because 000$ & \\
\hline 造芩 & $\dot{U} \vec{N} \vec{N}$ & 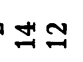 & ొొః & $\because$ 赵赵m & $+\infty M N$ \\
\hline 密 & 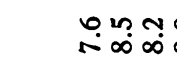 & : & স্ & 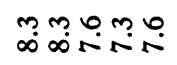 & $\stackrel{0}{-\infty} 0.0$ \\
\hline$\sum_{\bar{z}}^{\infty}$ & $1+1$ & +1 & $1++++$ & $++11+$ & $++t$ \\
\hline$\frac{\nabla}{0}$ & 111 & 11 & １１１芯芯卢 & $\begin{array}{lllll}1 & 1 & 1 & 1 & 1\end{array}$ & $\begin{array}{llll}1 & 1 & 1 & 1\end{array}$ \\
\hline 芦 & 111 & $\dot{H}+$ & +11111 & $11+++$ & $\begin{array}{llll}1 & 1 & 1 & 1\end{array}$ \\
\hline 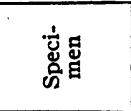 & 营 & ક્ळ & 莺 & 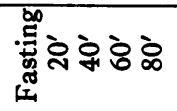 & 苞总就 \\
\hline 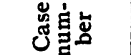 & ని & & 요 & $\vec{m}$ & กี \\
\hline
\end{tabular}


O. M. HELMER, PAUL J. FOUTS AND L. G. ZERFAS

\begin{tabular}{|c|c|c|c|c|}
\hline 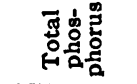 & 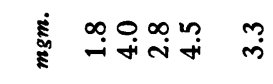 & 어 & 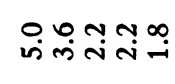 & $\stackrel{10}{0} \stackrel{0}{+\infty}$ \\
\hline 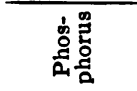 & 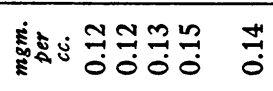 & 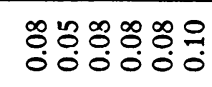 & 웅궁융중융 & $5 \div$ \\
\hline 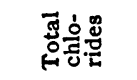 & 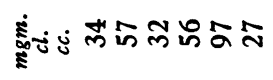 & 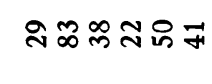 & 央京ニュニ & \\
\hline 施苞 & 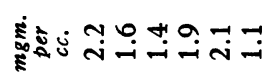 & 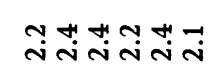 & 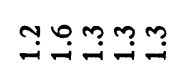 & \\
\hline 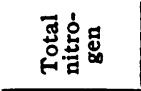 & है & & 이류요 & \\
\hline 室: & : & & 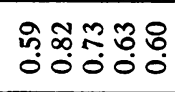 & \\
\hline 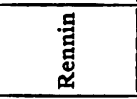 & 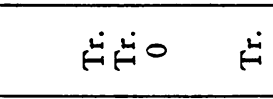 & 000000 & 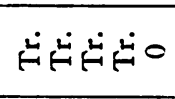 & 0 \\
\hline 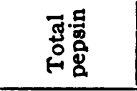 & 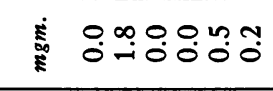 & 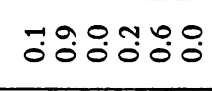 & 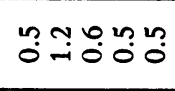 & 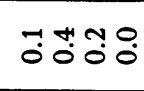 \\
\hline 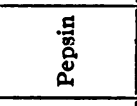 & : & 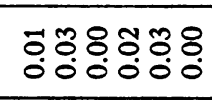 & 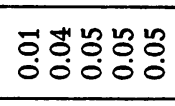 & 훙ㅇㅇㅇㅇㅠ \\
\hline 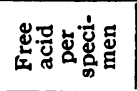 & ن) & 000000 & 00000 & 0000 \\
\hline 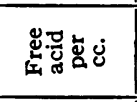 & $8: 000000$ & 000000 & 00000 & 0000 \\
\hline 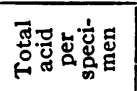 & 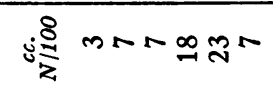 & & 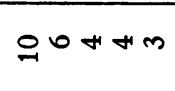 & 0000 \\
\hline 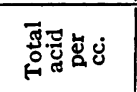 & 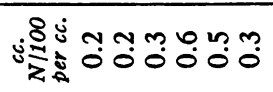 & & 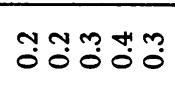 & $\because 000.0$ \\
\hline 安苜 & 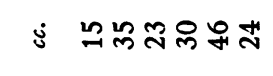 & 꿈으으증 & 욤유믐 & Hopom \\
\hline 空 & 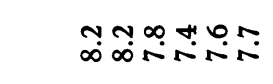 & 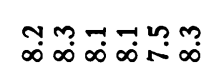 & 잉요 & 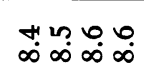 \\
\hline 总 & 111111111 & $\begin{array}{l}++++ \\
++++\end{array}$ & 1111111 & $+1++$ \\
\hline$\frac{\vec{\sigma}}{\dot{m}}$ & 1111111 & 1111111 & 1111111 & $111+$ \\
\hline$\cong$ & $++1 \stackrel{+}{+}+\dot{+}$ & 111111111 & 11111 & 11111 \\
\hline 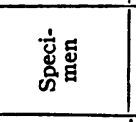 & 总点 & 兽 & 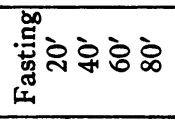 & 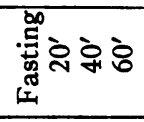 \\
\hline هั & $m$ & $\ddot{m}$ & $m$ & i \\
\hline
\end{tabular}




\begin{tabular}{|c|c|c|c|c|c|}
\hline 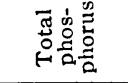 & 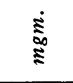 & $\stackrel{0}{-1}$ & $\vec{m} \stackrel{\infty}{i}$ & $\stackrel{0}{i} \stackrel{n}{0}$ & \\
\hline 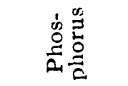 & 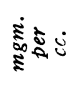 & $=80 \%$ & $\frac{3}{0} \div$ & : & \\
\hline 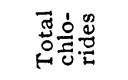 & نे & 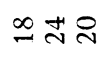 & 岕 & & \\
\hline 完: & 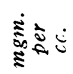 & $\stackrel{0}{i} \stackrel{0}{-}$ & $\stackrel{4}{\longrightarrow}$ & & \\
\hline 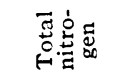 & 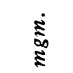 & $r=\infty$ & $\approx$ & & \\
\hline 䓂范 & نें & 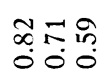 & $\vec{E} \overline{0}$ & & \\
\hline 音 & & 000 & $00 \quad \dot{H}$ & 0 & 000000 \\
\hline 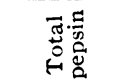 & $\begin{array}{l}\dot{\bar{\Delta}} \\
\text { है }\end{array}$ & $\overrightarrow{0}: 0 . \ln$ & : & $\because \div 0: 0$ & 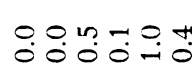 \\
\hline 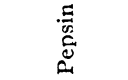 & 㝘ま & 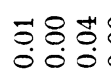 & : & $\begin{array}{l}8008 \\
0000\end{array}$ & 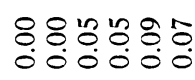 \\
\hline 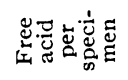 & $\dot{ن} \frac{8}{z}$ & 000 & $=0000000$ & 0000 & 000000 \\
\hline 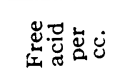 & ن & 000 & $=0000000$ & 0000 & 000000 \\
\hline  & $\dot{4} \frac{8}{z}$ & & & & \\
\hline نَ & ن & & & & \\
\hline 市芩 & ن & $a \pm$ & $\ddot{\sim} \approx m \operatorname{nn} a 0$ & $n=\infty N$ & $\sim \infty \cong \sim \cong 0$ \\
\hline ta & & 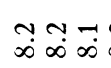 & 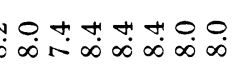 & بִ & ים \\
\hline$\stackrel{n}{\stackrel{n}{\Xi}}$ & & ++1 & $\begin{array}{lllllllll}1 & 1 & 1 & 1 & 1 & 1 & 1\end{array}$ & $1_{+}^{+}+$ & $\begin{array}{l}+++++ \\
++\end{array}$ \\
\hline$\frac{\overline{0}}{0}$ & & $1 \dot{\vec{H}}$ & 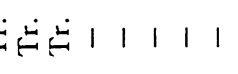 & $\begin{array}{llll}1 & 1 & 1 & 1\end{array}$ & $1111+1$ \\
\hline$\cong$ & & 111 & 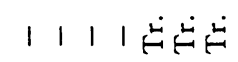 & 1111 & $11 \stackrel{\dot{H}}{\uplus}+\dot{\uplus}$ \\
\hline 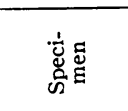 & & 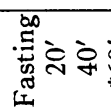 & bे & 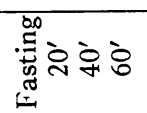 & $\underbrace{\infty}_{\substack{0 \\
0}}$ \\
\hline 总葟岂 & & $\hat{m}$ & $\infty$ & बे & 욱 \\
\hline
\end{tabular}


O. M. HELMER, PAUL J. FOUTS AND L. G. ZERFAS

\begin{tabular}{|c|c|c|c|c|}
\hline  & : & مْ & & 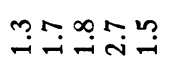 \\
\hline 它竞 & 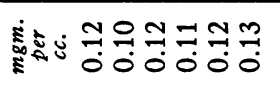 & 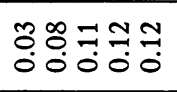 & & 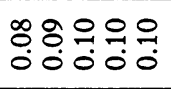 \\
\hline 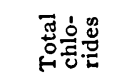 & 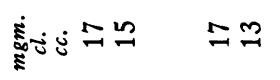 & 윰유 & & 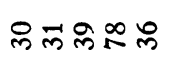 \\
\hline 递苞 & 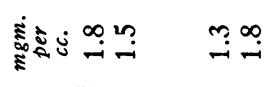 & 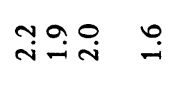 & & 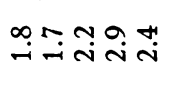 \\
\hline 至宫焉 & हैं & $-20+$ & & 으귝구 \\
\hline 产若 & 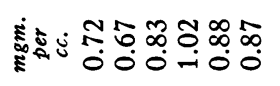 & 유융 & & 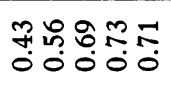 \\
\hline 竞 & 00000 & 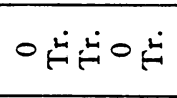 & & 捳芦芦出 \\
\hline 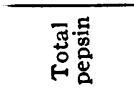 & 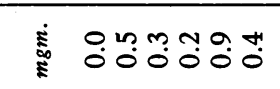 & : & $\overrightarrow{0}: \stackrel{\infty}{0}$ & 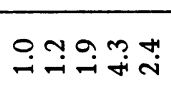 \\
\hline 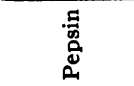 & 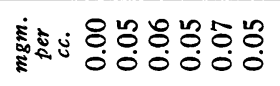 & :.8:8: & mot & 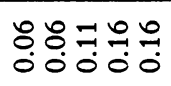 \\
\hline 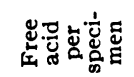 & $: \frac{8}{z} 000000$ & 00000 & 0000 & 00000 \\
\hline ن & ن: & 00000 & 0000 & 00000 \\
\hline 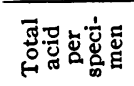 & is & & & oOngo \\
\hline نं & ن⿺辶 & & & $\because 0: \div 000$ \\
\hline 官苛 & ¿ $a$ gnm & $=\pi m a$ & mon & 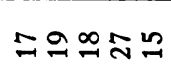 \\
\hline 密 & Oํ. & 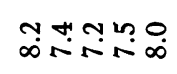 & 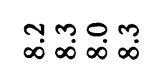 & ஸุ \\
\hline$\sum_{\bar{z}}^{\infty}$ & $\begin{array}{llllll}1 & 1 & 1 & 1 & 1 & 1\end{array}$ & ++++ & $\begin{array}{l}++++ \\
++++\end{array}$ & +++++ \\
\hline$\frac{\square}{0}$ & $111+11$ & 11111 & $1+1+$ & $\begin{array}{lllll}1 & 1 & 1 & 1 & 1\end{array}$ \\
\hline 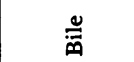 & $\begin{array}{llllll}1 & 1 & 1 & 1 & 1 & 1\end{array}$ & 11111 & 1111 & $\begin{array}{lllll}1 & 1 & 1 & 1 & 1\end{array}$ \\
\hline $\begin{array}{l}\text { 它: } \\
\text { 遌 }\end{array}$ & 营 & 莺兽 & 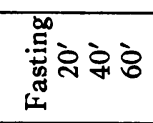 & 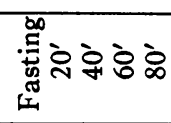 \\
\hline 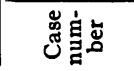 & $F$ & $\mathcal{F}$ & $\dddot{F}$ &  \\
\hline
\end{tabular}




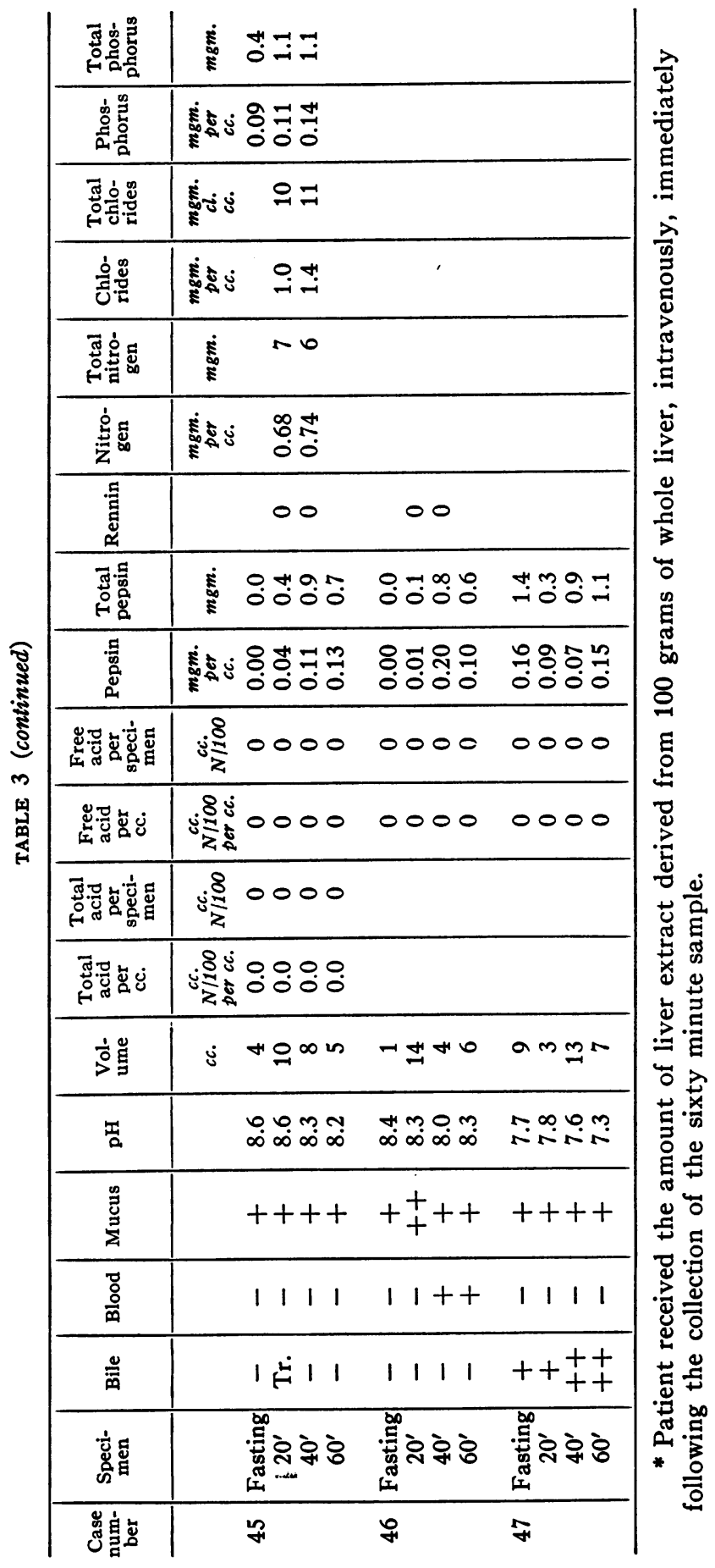


Volume. Histamine stimulation caused an increase in volume over the fasting sample in twenty-five of the forty-seven cases examined. At the height of secretion, the volume varied from 4 to $83 \mathrm{cc}$., with an average of $22 \mathrm{cc}$. The total volume secreted in sixty minutes varied

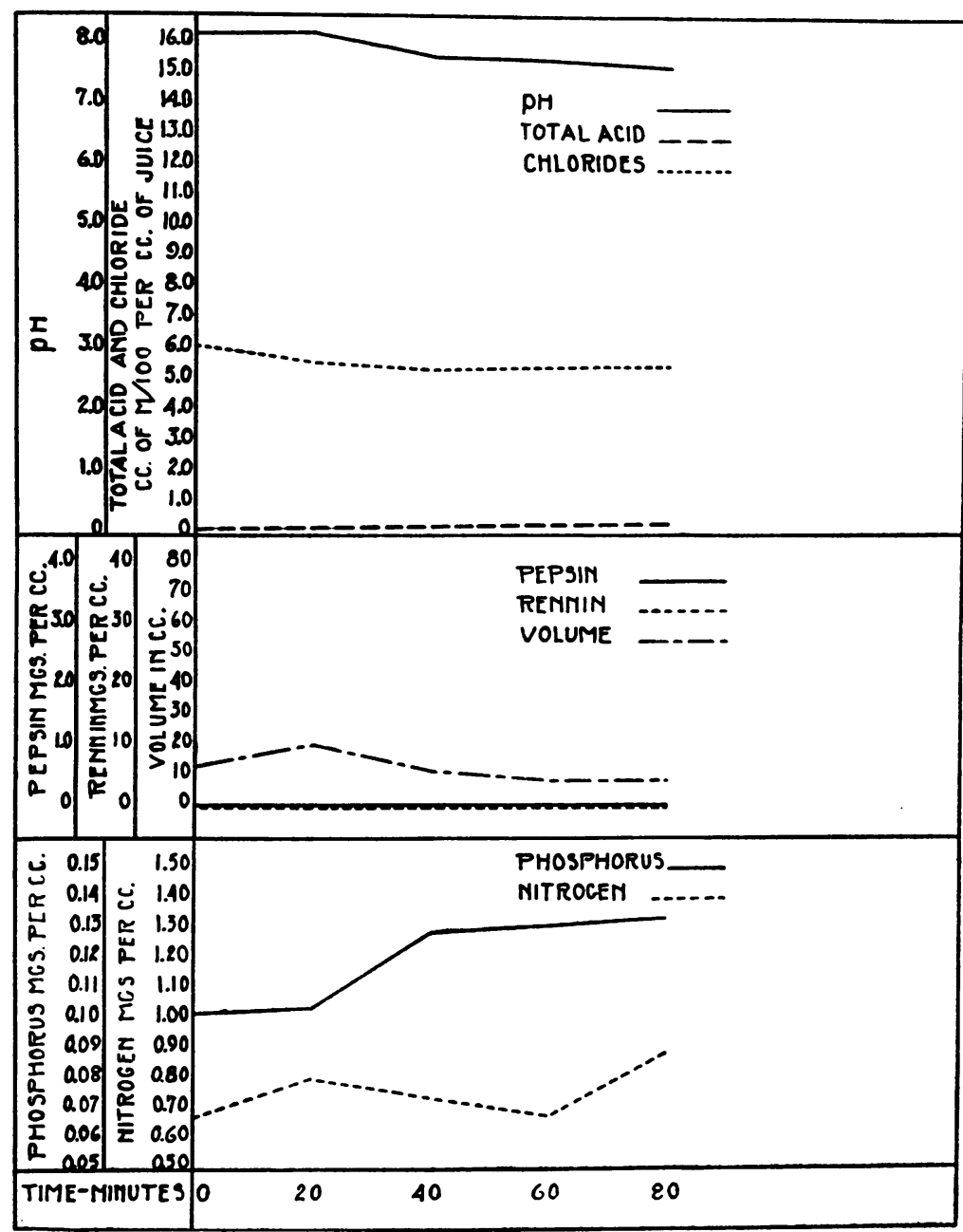

Average Values from Gastric Juice of Forty-seven Patients with Pernicious Anemia, Following the Subcutaneous Injection of 0.5 Mgm. OF Histamine Hydrochloride

from 9 to $193 \mathrm{cc}$., with an average of $41 \mathrm{cc}$. Following the injection of liver extract there was an increase in volume in all but one of fourteen cases.

The patients who had lower red blood cell counts tended to have smaller volumes than those having higher counts. Seven of eight patients 
with red blood cell counts below three million had sixty minute volumes of less than $25 \mathrm{cc}$. Patients with early or no central nervous system involvement more often had a greater volume of gastric juice than those with moderate or advanced central nervous system involvement. It would seem that the patients with larger volumes more frequently had $\mathrm{pH}$ values of the gastric juice that decreased after histamine stimulation, although the volume had no relation to the exact $\mathrm{pH}$ value.

The percentage of pepsin in the individual samples bore little or no relationship to the volume; however, the patients with larger total volumes tended to have the higher total pepsin values, although a few of the patients with the highest volumes had the smallest amounts of pepsin. The amounts of rennin present seemed to have no relation to the volume of the sample. Contrary to expectations, the percentage of phosphorus was higher in the samples with the greater volumes. Likewise, in the cases with greater volumes, there was a higher percentage of cases in which there was an increase in phosphorus values over the fasting. The samples having larger volumes usually had a higher percentage of chlorides. The amount of nitrogen in a sample bore no relationship to the volume of the sample.

Acidity. In all samples there was an absence of free hydrochloric acid and the $\mathrm{pH}$ varied from 6.9 to 8.6. Only five of the forty-seven patients had not received liver extract medication previous to the gastric analysis. The length of time the others had been on liver extract medication varied from a few months to five years. This continued absence of free acid in the gastric juice of patients with pernicious anemia, after oral liver therapy, is very well known, and it is interesting to note that the seventeen patients who have received liver extract by injection for from one to eight months still have an achylia gastrica. The amount of total acid excreted in sixty minutes varied between 0 and $30 \mathrm{cc}$. N/100. In only fourteen cases was there a measurable drop in $\mathrm{pH}$ value after histamine stimulation. After liver extract injection there was no constant change in $\mathrm{pH}$ value. The presence of bile in the samples caused an increase in the amount of total acid present and a lowering of the $\mathrm{pH}$. There was no relation between the amount of total acid present or the height of the $\mathrm{pH}$ and the clinical condition of the patient, except that only one of eight patients with red blood cell counts below 3.0 million had a $\mathrm{pH}$ value below 7.5 in any sample. However, in the fourteen instances in which the $\mathrm{pH}$ of the gastric juice decreased after histamine, nine of the patients had only early or no central nervous system involvement. The height of the $\mathrm{pH}$ bore no relation to the percentage of pepsin, rennin, phosphorus, chlorides, or nitrogen in the samples; but, in the cases with a decrease in $\mathrm{pH}$ value after histamine stimulation, there was a greater percentage with an increase in phosphorus, chlorides, and nitrogen.

Pepsin. The pepsin in the gastric juice was either totally absent or 
present in essentially negligible amounts, varying between 0.0 and 0.20 mgm. per cc., with an average of $0.04 \mathrm{mgm}$. The total amount of pepsin secreted in sixty minutes varied between 0.0 and $7.4 \mathrm{mgm}$., with an average of 1.8. There was a slight increase in the amount of pepsin after histamine stimulation in thirty-three of the forty-seven cases examined. The injection of liver extract caused an increase in pepsin in seven of fourteen cases. The clinical condition of the patient did not seem to show any relationship to the percentage of pepsin present in the samples. Likewise, the amount of pepsin showed no relation to the height of $\mathrm{pH}$, amount of phosphorus, chlorides, or nitrogen, although thirteen of seventeen cases with an increase in nitrogen after histamine also had an increase in pepsin. A trace of bile in the specimen occasionally caused a slight increase in peptic activity, but even then the amount of pepsin found was not over $0.17 \mathrm{mgm}$. per cc. Occasionally, when bile was present, there was a total absence of peptic activity.

Rennin. The amount of rennin present varied from 0.0 to $0.19 \mathrm{mgm}$. per cc., with an average of 0.013 . The total amount of rennin secreted in sixty minutes varied from 0 to $4,0 \mathrm{mgm}$. (average $0.42 \mathrm{mgm}$.). Because of the very small amounts of rennin present - the maximum in the patients with pernicious anemia being only $1 / 350$ th of the maximum in the normals -it was decided to express the rennin in Chart II as either being present or absent. The presence of bile in the sample usually caused an increase in amount of rennin, but again there occasionally was no rennin in samples containing bile. There seemed to be no relation between the amount of rennin in the gastric sample and the clinical condition of the patient or the $\mathrm{pH}$ value, volume, amount of phosphorus, nitrogen, or chlorides. The determination of rennin was a good check on the pepsin determination, as there was always a proportionately greater decrease in the amount of rennin when there was a decrease in pepsin.

Nitrogen. The amount of nitrogen present was definitely high in all samples. The presence of bile in the samples caused a great increase in the amount of nitrogen, and, therefore, the nitrogen determinations were of no value in samples containing bile. The amount of nitrogen present in the samples of gastric juice had no relation to the clinical condition of the patient. An increase in the concentration of nitrogen was noted in six of eight patients who received liver extract intravenously. The amount of nitrogen present showed no relationship to $\mathrm{pH}$ values or to the amounts of phosphorus, chlorides, pepsin, or rennin.

Chloride. The chloride values were definitely low, but they did not seem to be as low as would be expected with a total absence of free hydrochloric acid-a few high values, obtained in patients with achylia, were greater than some of the lower values obtained in normal persons. There was an increase in chlorides after histamine stimulation in only nine of twenty-five cases. As has been shown above, there was a tendency 
for specimens with larger volumes to have higher percentages of chlorides. There was no correlation between the amounts of chlorides and the $\mathrm{pH}$, amounts of nitrogen, pepsin, rennin, and phosphorus in the samples.

Phosphorus. The amount of phosphorus present was greatly increased in nearly all specimens. In eighteen of twenty-five cases the phosphorus value increased after histamine stimulation. As has been shown, the specimens with larger volumes more of ten had higher phosphorus values, and a higher percentage of the specimens with larger volumes had an increase in phosphorus values after histamine stimulation. There was no correlation between the phosphorus values and the $\mathrm{pH}$, amounts of nitrogen, chlorides, pepsin, or rennin.

It is evident from these findings that, while in normal persons there is a direct relationship between the free acid, total acid, chlorides, phosphorus, and nitrogen in the gastric juice after histamine stimulation, there is absolutely no relationship between these substances in the gastric juice of patients with pernicious anemia.

Gastric juice findings in relation to maintenance dosage of liver extract

All of the forty-seven patients in this group were clinically and hematologically typical of pernicious anemia, and either had had or subsequently have had good responses to liver extract administered by mouth or by injection. The length of time that these patients have been on liver extract has varied from 0 to 5 years. Twenty-one of the patients have been followed at monthly intervals by this department for from over one to five years, and the remaining patients for only a few months. Seventeen were in relapse (either mild or severe) at the time the analyses were made. The red blood cell counts varied from 0.5 million to 6.0 million and the hemoglobin percentages from 13 to 115 per cent. All degrees of central nervous system involvement were represented-from the very slightest to the most advanced stage where the patient was completely bedridden. In this group of patients, some were able to maintain their blood at normal levels on a very small daily amount of potent liver extract by mouth, while others were not able to do this on large amounts and required liver extract by injection.

An attempt was made to determine whether the quantity or quality of the gastric juice bore any relation to the amount of liver extract required to maintain the blood at normal levels. There were thirty-one patients in the group who gave a history of having taken liver extract for a sufficient period of time to be considered. Six of the thirty-one were in relapse at the time the gastric analyses were taken. Since it has been shown that patients with a low red blood cell count have smaller amounts of gastric juice than those with a high red blood cell count, it is difficult to correlate the gastric juice findings of the patients in relapse and the maintenance dosage; therefore these patients were excluded. 
It would seem that patients with larger volumes of gastric juice, after histamine stimulation, more often were able to maintain the blood at normal levels on smaller amounts of liver extract. However, all but two of the sixteen patients requiring more than the amount of liver extract derived from 300 grams of whole liver daily had one or more complications, such as: moderate to advanced central nervous system involvement, generalized arteriosclerosis, infections, non-infectious complications (other than arteriosclerosis), or chronic diarrhea. Such complications, in themselves, have been shown by Beebe and Lewis (17) and by Fouts and Zerfas (18) to be present in a high percentage of patients requiring large daily amounts of potent liver extract. These facts make it difficult to correlate the gastric juice findings with the maintenance dosage of liver extract; however, it is interesting to note that, of nine patients with no complications, six had large total volumes of gastric juice and only one required more than 300 grams of liver daily, while one of the three patients having small total volumes required more than that amount of liver.

The amount of pepsin per cc. of gastric juice had no bearing on the amount of liver extract required by a patient. Since the total amount of pepsin secreted in sixty minutes varied approximately as did the volume, the total amount of pepsin bore the same relationship to maintenance dosage of liver extract as did the volume. The amount of rennin, likewise, had no relation to the maintenance dose of liver extract. The $\mathrm{pH}$ value, amount of total acid, amount of phosphorus, chlorides, and nitrogen had no correlation with the amount of liver extract required to maintain the blood at normal levels.

From these facts it is evident that the quantity and quality (as tested) of the gastric juice have little or no relationship to the maintenance dosage of liver extract. The only positive finding was that larger volumes and larger total amounts of pepsin have a questionable correlation with the maintenance dosage. Davies (3) found that two of his patients, who were able to maintain their blood at normal levels with very small amounts of liver extract, had large amounts of pepsin. We were not able to find appreciable amounts of pepsin or rennin in any of the fortyseven cases of pernicious anemia examined. Also, we could not find any correlation between the small variations in the amount of pepsin and the maintenance dosage of liver extract. We were unable to test the gastric juice for the presence of, or for the quantitative changes in, the amounts of intrinsic factor of Castle. If there are no qualitative or quantitative differences in the proteolytic enzymes of the small intestines of patients with pernicious anemia or in the amounts of the intrinsic factor, it is evident that the ability of such patients to absorb the active principle contained in liver extract largely governs the amount of liver extract required to maintain the blood at normal levels, when the extract is 
administered orally. Castle (19) has suggested that there is a defective absorption of liver extract in some patients having pernicious anemia and further suggests that the inability to absorb the active principle from food is of etiological importance in the disease.

\section{CONCLUSIONS}

1. The gastric juice of forty-seven patients with pernicious anemia and of ten normal persons, after histamine stimulation, has been studied.

2. The complete dysfunction of the gastric glands in patients with pernicious anemia was demonstrated by the following facts: (a) Small volumes of juice containing large amounts of mucus; $(b)$ Absence of free hydrochloric acid, with an actual alkaline reaction; $(c)$ Practical absence of the gastric enzymes, pepsin and rennin; $(d)$ Low chloride concentration; (e) High nitrogen and phosphorus values; $(f)$ No relation between total acid, $\mathrm{pH}$, chlorides, nitrogen, and phosphorus as found in normal controls.

3. The quality and quantity of the gastric juice of patients with pernicious anemia has little or no relationship to the maintenance dosage of liver extract.

\section{BIBLIOGRAPHY}

1. Michaelis, L., Deutsche med. Wchnschr., 1918, xliv, 685. Die Bestimmung und Bedeutung der Fermente im Magensaft.

2. Polland, W. Scott, and Bloomfield, A. L., J. Clin. Invest., 1931, ix, 107. The Diagnostic Value of Determinations of Pepsin in Gastric Juice.

3. Davies, Daniel T., Quart. J. Med., 1931, xxiv, 447. Studies on Achlorhydria and Anemia.

4. Brown, J. H., J. Lab. and Clin. Med., 1924, ix, 239. The Colorimetric Determination of the Hydrogen Ion Concentration of Small Amounts of Fluid.

5. Wilson, D. W., and Ball, E. G., J. Biol. Chem., 1928, lxxix, 221. A Study of the Estimation of Chloride in Blood and Serum.

6. Van Slyke, Donald D., J. Biol. Chem., 1927, 1xxi, 235. Gasometric Micro-Kjeldahl Determination of Nitrogen.

7. Fiske, C. H., and Subbarow, R., J. Biol. Chem., 1925, lxvi, 375. The Colorimetric Determination of Phosphorus.

8. Koch, F. C., and McMeekin, T. L., Personal Communication.

9. Polland, W. Scott, and Bloomfield, A. L., J. Clin. Invest., 1929, vii, 57. Quantitative Measurements of Pepsin in Gastric Juice Before and After Histamine Stimulation.

10. Gamble, James L., and McIver, M. A., Proc. Soc. Exper. Biol. and Med., 1926, xxiii, 439. Fixed Base in Gastric Juice.

11. Austin, J. H., and Gammon, G. D., J. Clin. Invest., 1931, x, 287. Gastric Secretion after Histamine: Sodium and Potassium Content and Pepsin Estimation.

12. Bulger, H. A., Stroud, C. M., and Heideman, M. L., J. Clin. Invest., 1928, v, 547. Studies of the Chemical Mechanism of Hydrochloric Acid Secretion. I. Electrolyte Variations in Human Gastric Juice.

13. Hoesch, K., Deutsches Arch. f. klin. Med., 1929, clxv, 201. Beiträge zur Mangenfunktion. I. Über die Phosphate im reinen Magensaft. 
14. Bolton, Charles, and Goodhart, Gordon W., J. Physiol., 1931, lxxiii, 115. The Variations in the Acidity of the Gastric Juice During Secretion.

15. Vineburg, Arthur M., and Babkin, B. P., Am. J. Physiol., 1931, xcvii, 69. Histamine and Pilocarpin in Relation to the Gastric Secretion.

16. Gilman, Alfred, and Cowgill, George R., Am. J. Physiol., 1931, xcvii, 124. Effect of Histamine upon the Secretion of Gastric Pepsin.

17. Beebe, Richard T., and Lewis, E. G. Eric, Am. J. Med. Sci., 1931, clxxxi, 796. The Maintenance Dose of Potent Material in Pernicious Anemia.

18. Fouts, Paul J., and Zerfas, L. G., Ann. Int. Med., In Press. Maintenance Dosage of Liver Extract in the Treatment of Pernicious Anemia.

19. Castle, William B., and Taylor, F. H. Laskey, J. Am. Med. Assoc., 1931, xcvi, 1198. Intravenous Use of Extract of Liver. 